\title{
Astérisque
}

\section{V.S. BUSLAEV}

GALINA PERELMAN

\section{On nonlinear scattering of states which are close to a soliton}

Astérisque, tome 210 (1992), p. 49-63

$<$ http://www.numdam.org/item?id=AST_1992_210_49_0>

(C) Société mathématique de France, 1992, tous droits réservés.

L'accès aux archives de la collection « Astérisque » (http://smf4.emath.fr/ Publications/Asterisque/) implique l'accord avec les conditions générales d'utilisation (http://www.numdam.org/conditions). Toute utilisation commerciale ou impression systématique est constitutive d'une infraction pénale. Toute copie ou impression de ce fichier doit contenir la présente mention de copyright.

\section{Numdam}

Article numérisé dans le cadre du programme Numérisation de documents anciens mathématiques http://www.numdam.org/ 


\section{ON NONLINEAR SCATTERING OF STATES \\ WHICH ARE CLOSE TO A SOLITON \\ V.S.Buslaev and G.S.Perelman}

\section{Solitons}

Consider the nonlinear Schroedinger equation

$$
i \psi_{t}=-\psi_{x x}+F\left(|\psi|^{2}\right) \psi, \psi=\psi(x, t) \in \mathbf{C},
$$

$x, t \in \mathbf{R}$. Assume that

i) $F$ is a given smooth $\left(\in C^{\infty}\right)$ real function bounded from below,

ii) the point $\xi=0$ is a (sufficiently strong) root of the function $F$ :

$$
F(\xi)=F_{1} \xi^{p}(1+O(\xi)), p>0 .
$$

Further consider the function

$$
U(\phi, \alpha)=-\frac{1}{8} \alpha^{2} \phi^{2}-\frac{1}{2} \int_{0}^{\phi^{2}} F(\xi) d \xi
$$

If $\alpha \neq 0$ this function is negative for sufficiently small $\phi$. The next assumption on $\mathrm{F}$ will be given in a sligtly implicit, but absolutely elementary form:

iii)for $\alpha$ from some interval, $\alpha \in A \subset \mathbf{R}_{+}$, the function $\phi \rightarrow U(\phi, \alpha)$ has a positive root; if $\phi_{0}\left(=\phi_{0}(\alpha)\right)$ is the smallest positive root then $U_{\phi}\left(\phi_{0}, \alpha\right)>0$.

Under all these assumptions there exists the unique even positive solution $y \rightarrow \phi(y)$ of the equation

$$
\phi_{y y}=-U_{\phi}=\frac{1}{4} \alpha^{2} \phi+F\left(\phi^{2}\right) \phi
$$

vanishing at infinity. More precisely

$$
\phi=\phi(y \mid \alpha) \sim \phi_{\infty} \exp \left(-\frac{1}{2} \alpha|y|\right), y \rightarrow \infty .
$$

The following functions of $x$ can be called the soliton states:

$$
w(x \mid \sigma)=\exp \left(-i \beta+i \frac{1}{2} v x\right) \phi(x-b \mid \alpha),
$$


here

$$
\sigma=(\beta, \omega, b, v), \omega=\frac{1}{4}\left(v^{2}-\alpha^{2}\right)
$$

$\beta, \omega, b, v \in \mathbf{R}, \alpha \in A$. The set of the allowable $\sigma$ will be denoted by $\Sigma$. If $\sigma$ is a solution of the Hamiltonian system:

$$
\beta^{\prime}=\omega, \omega^{\prime}=0, b^{\prime}=v, v^{\prime}=0 .
$$

the function $w(x \mid \sigma(t))$ is a solution of the equation (1.1) called the soliton.

\section{The linearization of equation (1.1)}

Consider the linearization of the equation (1.1) on the soliton $w(x \mid \sigma(t))$ :

$$
i \chi_{t}=-\chi_{x x}+F\left(|w|^{2}\right) \chi+F^{\prime}\left(|w|^{2}\right) w(\bar{w} \chi+w \bar{\chi})
$$

Instead of $\chi$ introduce the function $f$ :

$$
\chi(x, t)=\exp (i \Phi) f(y, t), \Phi=-\beta(t)+\frac{1}{2} v x, y=x-b(t)
$$

The function $f$ obeys the following equation:

$$
i f_{t}=L(\alpha) f
$$

where

$$
L(\alpha) f=-f_{y y}+\frac{1}{4} \alpha^{2} f+F\left(\phi^{2}\right) f+F^{\prime}\left(\phi^{2}\right) \phi^{2}(f+\bar{f}),
$$

$\phi=\phi(y \mid \alpha)$. Equation (2.3) is only a real-linear equation. Introduce its complexification:

$$
\begin{gathered}
i \vec{f}_{t}=H(\alpha) \vec{f}, \vec{f}=\left(\begin{array}{c}
f \\
\vec{f}
\end{array}\right), \\
H(\alpha)=H_{0}(\alpha)+V(\alpha), H_{0}(\alpha)=\left(-\partial_{y}^{2}+\frac{1}{4} \alpha^{2}\right) \sigma_{3}, \\
V(\alpha)=\left[F\left(\phi^{2}\right)+F^{\prime}\left(\phi^{2}\right) \phi^{2}\right] \sigma_{3}+i F^{\prime}\left(\phi^{2}\right) \phi^{2} \sigma_{2},
\end{gathered}
$$

$\sigma_{2}, \sigma_{3}$ are the standard Pauli matrices:

$$
\sigma_{2}=\left(\begin{array}{cc}
0 & -i \\
i & 0
\end{array}\right), \sigma_{3}=\left(\begin{array}{cc}
1 & 0 \\
0 & -1
\end{array}\right)
$$




\section{Properties of the operator $H(\alpha)$}

The operator $H(\alpha)$ can be treated as a linear operator in $\mathbf{L}_{2}\left(\mathbf{R} \rightarrow \mathbf{C}^{2}\right)$. Define it on the domain where $H_{0}(\alpha)$ is self-adjoint. It possesses the properties:

$$
\sigma_{3} H=H^{*} \sigma_{3}, \sigma_{2} H=-H^{*} \sigma_{2}, \sigma_{1} H=-H \sigma_{1} .
$$

As a result the spectrum of $H$ is invariant with respect to the following transformations: $E \rightarrow \bar{E}, E \rightarrow-E$.

The continuous spectrum consists of two half-axis $\left[E_{0}, \infty\right)$ and $\left(-\infty,-E_{0}\right]$, $E_{0}=\frac{1}{4} \alpha^{2}$. Its multiplicity is equal to 2 .

Owing to the exponential decay of the potential term $V(\alpha)$ at infinity the discrete spectrum of $H(\alpha)$ contains only a finite number of eigenvalues and the corresponding root subspaces have only finite dimension.

The point $E=0$ is always a point of the discrete spectrum. One can indicate two eigenfunctions

$$
\vec{\xi}_{1}=\left(\begin{array}{c}
u_{1} \\
\bar{u}_{1}
\end{array}\right), \vec{\xi}_{3}=\left(\begin{array}{c}
u_{3} \\
\bar{u}_{3}
\end{array}\right)
$$

where

$$
u_{1}=-i \phi(y \mid \alpha), u_{3}=-\phi_{y}
$$

and two adjoint functions:

$$
\vec{\xi}_{2}=\left(\begin{array}{c}
u_{2} \\
\bar{u}_{2}
\end{array}\right), \vec{\xi}_{4}=\left(\begin{array}{c}
u_{4} \\
\bar{u}_{4}
\end{array}\right)
$$

where

$$
u_{2}=-\frac{2}{\alpha} \phi_{\alpha}, u_{4}=\frac{i}{2} y \phi
$$

They obey the relations:

$$
H \vec{\xi}_{1}=H \vec{\xi}_{3}=0, H \vec{\xi}_{2}=i \vec{\xi}_{1}, H \vec{\xi}_{4}=i \vec{\xi}_{3} .
$$

Actually, the spectrum of $H(\alpha)$ can lie only in the real axis and in the imaginary axis of the $E$-plane, see [We2], for example. It is known also that the spectrum of $H(\alpha)$ is real and the root subspace corresponding to the point $E=0$ is generated by the vectors $\vec{\xi}_{1}, \vec{\xi}_{2}, \vec{\xi}_{3}, \vec{\xi}_{4}$ if and only if

$$
\partial_{\alpha}\|\phi\|^{2}>0 \text {. }
$$

Consider the resolvent $R(E)=(H-E)^{-1}$. Its kernel $R\left(y, y^{\prime} \mid E\right)$ is an analytic function in the extended $E$-plane: it admits an analytic continuation through the continuous spectrum as a meromorphic function. The resolvent kernel goes to infinity when $E$ tends to the branch points $\mp E_{0}$ if the equation $H(\alpha) \psi=\mp E_{0} \psi$, treated as a differential equation, has nontrivial solutions bounded at infinity. In this case the points $\mp E_{0}$ will be called resonances. 


\section{Nonlinear equation}

Consider the Cauchy problem for equation (1.1) with the initial data

$$
\psi(x, 0)=\psi_{0}(x),
$$

where $\psi_{0} \in H^{1}, H^{1}$ is the standard Sobolev space with the norm:

$$
\|f\|_{H^{1}}^{2}=\|f\|_{2}^{2}+\left\|f^{\prime}\right\|_{2}^{2} .
$$

The problem has a solution $\psi=\psi(x, t)$ which belongs to $H^{1}$ with respect to $x$ for each $t$, moreover $\psi \in C\left(\mathbf{R} \rightarrow H^{1}\right)$. Any such solution $\psi$ obeys two conservation laws:

$$
\int|\psi(x, t)|^{2} d x=\text { const }, \int\left[\left|\psi_{x}(x, t)\right|^{2}+U(|\psi(x, t)|)\right] d x=\text { const },
$$

where $U$ is the function (1.3). The second formula (4.3) leads to the following estimate:

$$
\|\psi(\cdot, t)\|_{H^{1}} \leq c\left(\left\|\psi_{0}\right\|_{H^{1}}\right)\left\|\psi_{0}\right\|_{H^{1}}
$$

here $c=\mathbf{R}_{+} \rightarrow \mathbf{R}_{+}$is a smooth function. If in addition $\psi_{0}$ has the finite norm: $\left\|(1+|x|) \psi_{0}\right\|_{2}<\infty$, the solution $\psi$ also has the finite, but growing in time, similar norm:

$$
\|(1+|x|) \psi(x, t)\|_{2} \leq c\left(\left\|\psi_{0}\right\|_{H^{1}}\right)\left[\|(1+|x|) \psi\|_{2}+t\left\|\psi_{0}\right\|_{H^{1}}\right] .
$$

\section{Theorem}

Let $\sigma_{0}=\left(\beta_{0}, \omega_{0}, b_{0}, v_{0}\right) \in \Sigma, \omega_{0}=\frac{1}{4}\left(v_{0}^{2}-\alpha_{0}^{2}\right)$. Consider the Cauchy problem for equation (1.1) with the initial data:

$$
\psi_{0}(x)=w\left(x \mid \sigma_{0}\right)+\chi_{0}(x) .
$$

Our aim is to describe the asymptotic behavior of the solution $\psi$ as $t \rightarrow \infty$. Assume that:

$T_{1}$ ) the norm

$$
N=\left\|\left(1+x^{2}\right) \chi_{0}\right\|_{2}+\left\|\chi_{0}^{\prime}\right\|_{2}
$$

is sufficiently small;

$\left.T_{2}\right) E=0$ is the only point of the discrete spectrum of $H\left(\alpha_{0}\right)$ and the dimension of the corresponding root subspace is equal to 4 ; 
$\left.T_{3}\right)$ the points $\mp E_{0}$ are not resonances;

$T_{4}$ ) the function $F$ is a polynomial ${ }^{1}$ and $p \geq 4$.

Then there exist $\sigma_{+} \in \Sigma$ and $f_{+} \in \mathbf{L}_{2} \cap \mathbf{L}_{\infty}$ such that

$$
\psi=w\left(\cdot, \sigma_{+}(t)\right)+\exp \left(-i l_{0} t\right)+o(1)
$$

as $t \rightarrow \infty$. In this formula: $\sigma_{+}(t)$ is the trajectory of the system (1.8) with the initial data $\sigma_{+}(0)=\sigma_{+} ; l_{0}=-\partial_{x}^{2} ; o(1)$ is meant $\mathbf{L}_{2}$-norm. Moreover $\sigma_{+}$in $(5-3)$ is sufficiently close to $\sigma_{0}$ and $f_{+}$is sufficiently small.

It is worth to note that the operator $H(\alpha)$ possesses theses two properties $T_{2}$ ) and $\left.T_{3}\right)$ if $\alpha$ is sufficiently close to $\alpha_{0}$ and the operator $H\left(\alpha_{+}\right)$possesses both these properties naturally.

\section{Literature}

Of course, simple formulas (1.6-8) for the soliton are well known. But in many dimensional $x$-space the situation is quite different,see,for example, [Str2, Be-Li]. Properties of the spectrum of the operator $H(\alpha)$ were considered in [We2]. The Cauchy problem for equation (1.1) was considered in the space $H^{1}$ in $[\mathrm{G}-\mathrm{V}, \mathrm{K}]$ and in some other works. The Cauchy problem with the initial data of the form (5.1) was treated in [Sh-Str, Ca-Li, We1, We2]. The main result states that for the Cauchy data (5.1) the solution always remains in a small $H^{1}$-vicinity of the orbit generated by the trajectory $\sigma_{0}(t), \sigma_{0}(0)=\sigma_{0}$. As for the scattering behavior of the solution when $t \rightarrow \infty$, some series of works devoted to the scattering in the absence of bound states should be mentioned [Str1, G-V]. The only result which is close to formula (5.3) is contained in [Sof-We]. The authors of the work have considered the equation

$$
\begin{gathered}
i \psi_{t}=-\Delta \psi+\left[V(x)+\lambda|\psi|^{m-1}\right] \psi, \\
\psi=\psi(x, t), x \in \mathbf{R}^{n}, 1<m<\frac{n+2}{n-2}, n=2,3
\end{gathered}
$$

In this situation the soliton appears as the perturbation of an eigenfunction of the operator $\psi \rightarrow-\triangle \psi+V(x) \psi$, which is supposed to be unique and simple. The main difference between the theorem of [Sof-We] and our theorem is generated by the fact that in the first case the center of the soliton is stable. As a result our work containes some number of technical detailes which differ it from [Sof-We] although the main line is the same. However, it is worth to emphasize that this common mane line is also similar to the corresponding one in the investigation of asymptotic regimes for nonlinear parabolic equations,see[He].

\footnotetext{
${ }^{1}$ This assumption is not crucial, it is accepted only for the simplicity
} 


\section{Separation of motions}

Consider a trajectory $\sigma(t)=(\beta(t), \omega(t), b(t), v(t)) \in \Sigma$ which generally is not a solution of system (1.8).Consider the corresponding trajectory in the set of soliton states

$$
\begin{gathered}
w(x \mid \sigma(t))=\exp (i \Phi) \phi(y \mid \alpha), \\
\Phi=-\beta(t)+\frac{1}{2} v(t) x, y=x-b(t), \alpha=\alpha(t) .
\end{gathered}
$$

Write the solution $\psi$ of the Cauchy problem (1.1),(5.1) as the sum

$$
\psi(x, t)=w(x \mid \sigma(t))+\chi(x, t) .
$$

Instead of the equation (1.1) one can get a system for $\sigma$ and $\chi$ introducing some conditions on the splitting (7.2). Let $w_{\sigma}$ be the derivative of $w(x \mid \sigma)$ with respect to the parameter $\sigma$ and $w_{\sigma}(t)=w_{\sigma}(\cdot \mid \sigma(t))$. One will use the following condition to fix the splitting:

$$
\left\langle\vec{\chi}(t), \sigma_{3} \vec{w}_{\sigma}(t)\right\rangle=0,
$$

here $\langle\cdot, \cdot\rangle$ is the scalar product in the space $\mathbf{L}_{2}\left(\mathbf{R} \rightarrow \mathbf{C}^{2}\right)$. Note that

$$
\begin{gathered}
\vec{w}_{\beta}=\exp \left(i \sigma_{3} \Phi\right) \vec{\xi}_{1}, \vec{w}_{\omega}=\exp \left(i \sigma_{3} \Phi\right) \vec{\xi}_{2}, \\
\vec{w}_{b}=\exp \left(i \sigma_{3} \Phi\right) \vec{\xi}_{3}, \vec{w}_{v}=\exp \left(i \sigma_{3} \Phi\right)\left(\vec{\xi}_{4}-\frac{1}{2} b \vec{\xi}_{1}-\frac{1}{2} v \vec{\xi}_{2}\right) .
\end{gathered}
$$

So conditions (7.3) can be represented in the form:

$$
\left\langle\vec{f}(t), \sigma_{3} \vec{\xi}_{i}(t)\right\rangle=0,
$$

where

$$
\chi(x, t)=\exp (i \Phi) f(y, t), \vec{\xi}_{i}(t)=\vec{\xi}_{i}(y \mid \alpha(t)) .
$$

A different but an equivalent form of the splitting condition is

$$
i m\left(f(t), u_{i}(t)\right)=0 .
$$

The geometrical sense of $(7.3)=(7.6)=(7.8)$ is very simple: condition (7.6) implies that $\vec{f}(t)$ belongs to the subspace of the continuous spectrum of the operator $H(\alpha(t))$. Actually the condition leads to decomposition which is in accordance with the asymptotic behavior (5.3).

Write down the system for $\sigma$ and $\chi$ (or $f$ ) in more explicit form. Replace the set $\sigma=(\beta, \omega, b, v)$ by some other set of variables $(\gamma, \omega, c, v)$, where

$$
b=\int_{0}^{t} v(\tau) d \tau+c, \beta=\int_{0}^{t} \omega(\tau) d t+\gamma .
$$


In terms of new variables system (1.8) acquires the form:

$$
\gamma^{\prime}=0, \omega^{\prime}=0, c^{\prime}=0, v^{\prime}=0 .
$$

Rewrite equation (1.1) in terms of $\mathrm{f}$ :

$$
i f_{t}=L(\alpha) f+N(\phi, f)+l(\sigma) f+l(\sigma) \phi+i\left(\omega^{\prime}-\frac{1}{2} v v^{\prime}\right) \frac{2}{\alpha} \phi_{\alpha},
$$

where

$$
\begin{gathered}
N(\phi, f)=F\left(|\phi+f|^{2}\right)(\phi+f)-F\left(\phi^{2}\right) \phi-F\left(\phi^{2}\right) f \\
-F^{\prime}\left(\phi^{2}\right) \phi^{2}(f+\bar{f}), \\
l(\sigma)=\frac{1}{2} v^{\prime} y+i c^{\prime} \partial_{y}+\left(\frac{1}{2} b v^{\prime}-\gamma^{\prime}\right) .
\end{gathered}
$$

Consider the derivative of splitting condition in form (7.8) with respect to $t$ and substitute expression (7.11) for $f_{t}$ in the obtained relation. The result can be written down as follows:

$$
\left(A_{0}+A_{1}\right) \lambda=G
$$

where

$$
\lambda=\left(\gamma^{\prime}-\frac{1}{2} b v^{\prime}, \omega^{\prime}-\frac{1}{2} v v^{\prime}, c^{\prime}, v^{\prime}\right), A_{0}=\left\{i m\left(u_{i}, u_{j}\right)\right\}_{i, j=1}^{4},
$$

$(7.16)\left(A_{1} \lambda\right)_{j}=-r e\left(l(\sigma) f, u_{j}\right)-\frac{2}{\alpha}\left(\omega^{\prime}-\frac{1}{2} v v^{\prime}\right) i m\left(f, u_{j \alpha}\right), G=r e\left(N, u_{j}\right)$.

Obtain the explicit expression for the matrix $A_{0}$ :

$$
A_{0}=\left(\begin{array}{cccc}
0 & e & 0 & 0 \\
-e & 0 & 0 & 0 \\
0 & 0 & 0 & n \\
0 & 0 & -n & 0
\end{array}\right), n=-\frac{1}{4}\|\phi\|_{2}^{2}, e=\frac{4}{\alpha} \frac{d n}{d \alpha} .
$$

Under assumptions $\left.T_{2}\right), T_{3}$ )

$$
\operatorname{det} A_{0}\left(\alpha_{0} \neq 0\right. \text {, }
$$

see (3.7). If $\alpha(t)$ is close to $\alpha_{0}$ and $f(t)$ is sufficiently small (actually we are going to prove it), equation (7.14) can be used to estimate $\lambda$. Substituting $\lambda$ from (7.14) to the right side of (7.11) one obtains the system:

$$
\sigma_{t}=G_{1}(\sigma, f), i f_{t}=L(\alpha) f+N_{1}(\sigma, f) .
$$

Equation (7.14) is not a complete equivalent of conditions $(7.3)=(7.6)=(7.8)$. To get the equivalence one has to add to equation (7.14) condition $(7.3)=$ $(7.6)=(7.8)$ at the time-moment $t=0$ :

$$
\left\langle\vec{\chi}_{0}, \sigma_{3} \vec{w}_{\sigma}\left(\cdot \mid \sigma_{0}\right)\right\rangle=0 .
$$


Generally this condition is not satisfied by the given decomposition (5.1) of the initial data $\psi_{0}$. But if $\chi_{0}$ is sufficiently small it is possible to reconstruct decomposition (5.1) of the initial data $\psi_{0}$ in order to satisfy to condition (7.20)

In fact, one has to solve the equation:

$$
\left\langle\vec{\psi}_{0}-\operatorname{vecw}\left(\cdot \mid \sigma_{1}\right), \sigma_{3} \vec{w}_{\sigma}\left(\sigma_{1}\right)\right\rangle=0
$$

with respect to $\sigma_{1}$. Here $\psi_{0}$ should be given by (5.1) with sufficiently small $\chi_{0}$, see (5.2). The local solvability of (7.21) is guaranteed by the nondegeneration of the corresponding Jacobi matrix:

$$
-\left\langle\vec{w}_{\sigma_{i}}\left(\cdot \mid \sigma_{0}\right), \sigma_{3} \vec{w}_{\sigma_{j}}\left(\cdot \mid \sigma_{0}\right)\right\rangle=-2 i A_{0}\left(\alpha_{0}\right) .
$$

So one can assume that decomposition (5.1) obeys condition (7.20).

Since $\psi \in C\left(\mathbf{R} \rightarrow H^{1}\right)$ a little more general constructions show that condition (7.3) has to fulfil on some small time-interval. Some estimates which will be given in next sections, will show also that at the end of this time- interval the solution has the structure (5.1) with the small second term. It gives us the possibility to continue the constructions and to solve equation (7.3) for all $t \in\left[0, t_{1}\right]$.

\section{Reduction to a spectral problem}

Now one can describe the main line of the following constructions.

1)System (7.19) will be investigated on a large finite interval $t \in\left[0, t_{1}\right]$. In the end one will be able to consider the limit $t_{1} \rightarrow \infty$.

2) On the interval $\left[0, t_{1}\right]$ one can pick out the leading term of system (7.19) in the form:

$$
\sigma_{t}=0, i f_{t}=L(\alpha) f
$$

The first equation should be completed by more stable final data: $\sigma\left(t_{1}\right)=\sigma_{1}$ , with the undefinite for the moment values:

$$
\begin{gathered}
\sigma_{1}=\sigma\left(t_{1}\right), \sigma_{1}=\left(\beta_{1}, \omega_{1}, b_{1}, v_{1}\right), \\
\omega_{1}=\frac{1}{4}\left(v_{1}^{2}-\alpha_{1}^{2}\right), b_{1}=v_{1} t_{1}+c_{1}, \beta_{1}=\omega_{1} t_{1}+\gamma_{1} .
\end{gathered}
$$

Naturally now one has to put $L(\alpha)=L\left(\alpha_{1}\right)$. After that the second equation oppositely should be completed by the known initial data.

3)Rewrite full equation (7.11) in order to get the operator $L\left(\alpha_{1}\right)$ as the main term of the of the right side. Introduce the new function $g$ :

$$
\chi=\exp \left(i \Phi_{1}\right) g(z, t), \Phi_{1}=-\omega_{1} t-\gamma_{1}+\frac{1}{2} v_{1} x, z=x-v_{1} t-c_{1} .
$$


It obeys the equation:

$$
i g_{t}=L\left(\alpha_{1}\right) g+D(\sigma, g),
$$

and $\mathrm{D}$ is given by the formulas

$$
\begin{gathered}
D=D_{0}+D_{1}+D_{2}+D_{3}+D_{4} \\
D_{0}=\exp (-i \Delta)\left[l(\sigma) \phi(y \mid \alpha)+i \frac{2}{\alpha}\left(\omega^{\prime}-\frac{1}{2} v v^{\prime}\right) \phi_{\alpha}(y \mid \alpha)\right], \\
\Delta=\Phi_{1}-\Phi \\
D_{1}=\left[F\left(\phi^{2}(y \mid \alpha)+F^{\prime}\left(\phi^{2}(y \mid \alpha)\right) \phi^{2}(y \mid \alpha)\right] g-\right. \\
{\left[F\left(\phi^{2}\left(z \mid \alpha_{1}\right)\right)+F^{\prime}\left(\phi^{2}\left(z \mid \alpha_{1}\right) \phi^{2}\left(z \mid \alpha_{1}\right)\right] g\right.} \\
D_{2}=F^{\prime}\left(\phi^{2}(y \mid \alpha)\right) \phi^{2}(y \mid \alpha)[\exp (-2 i \Delta)-1] \bar{g} \\
D_{3}=\left[F^{\prime}\left(\phi^{2}(y \mid \alpha)\right) \phi^{2}(y \mid \alpha)-F^{\prime}\left(\phi^{2}\left(z \mid \alpha_{1}\right)\right) \phi^{2}\left(z \mid \alpha_{1}\right)\right] \bar{g} \\
D_{4}=\exp (-i \Delta) N(\phi(y \mid \alpha), \exp (i \Delta) g) .
\end{gathered}
$$

In order to investigate the long-time behavior of the solution of the second equation (8.1) and its full form (8.3) one has to separate the contributions of the discrete spectrum and of the continuous spectrum of the operator $L\left(\alpha_{1}\right)$, more precisely, of the operator $H\left(\alpha_{1}\right)$. Consider the representation

$$
\vec{g}=\vec{k}+\vec{h},
$$

where $\vec{k}$ and $\vec{h}$ are the indicated contributions. One can use condition (7.6) to express the component $\vec{k}$ in terms $\vec{h}$. Since

$$
\vec{k}=\sum_{i} \kappa_{i} \vec{\xi}_{i}\left(z \mid \alpha_{1}\right)
$$

condition (7.6) leads to the relation:

$$
\sum_{i} \kappa_{i}\left\langle\Lambda \vec{\xi}_{i}\left(z \mid \alpha_{1}\right), \sigma_{3} \vec{\xi}_{j}(y \mid \alpha)\right\rangle+\left\langle\Lambda \vec{h}, \sigma_{3} \vec{\xi}_{j}(y \mid \alpha)\right\rangle=0,
$$

where

$$
\Lambda=\left(\begin{array}{cl}
e^{i \Delta} & 0 \\
0 & e^{-i \Delta}
\end{array}\right)
$$

The main term of equation (8.7) is again defined by the matrix $A_{0}$ :

$$
\left\langle\vec{\xi}_{i}\left(z \mid \alpha_{1}\right), \sigma_{3} \vec{\xi}_{j}\left(y \mid \alpha_{1}\right)\right\rangle \sim-2 i A_{0}\left(\alpha_{1}\right) .
$$

At last for $\vec{h}$ one can write down the following integral representation (equation):

$$
\vec{h}=\exp \left(-i H_{1} t\right) \vec{h}_{0}-i \int_{0}^{t} \exp \left[-i H_{1}(t-\tau)\right] P_{1} \vec{D} d \tau \text {. }
$$


Here $P_{1}$ is the spectral projection operator on the subspace of the continuos spectrum of $H_{1}$ and

$$
\vec{h}_{0}=P_{1} \vec{g}_{0}, g_{0}(z)=\exp \left[i \gamma_{1}-i \frac{1}{2} v_{1}\left(z+c_{1}\right)\right] \chi_{0}\left(z+c_{1}\right) .
$$

The final form of the equations which are used in order to investigate the dynamical system on the interval $t \in\left[0, t_{1}\right]$ is given by relations $(7.14),(8.7),(8.10)$.

\section{Linear evolution}

Consider the operator $H=H(\alpha)$ with some fixed $\alpha$ and assume that $H$ satisfies conditions $\left.T_{2}\right), T_{3}$ ) (with $\alpha$ instead of $\alpha_{0}$ in them). Let $U(t)=\exp (-i H t)$ be the corresponding evolution operator and $P$ be the spectral projection operator on the subspace of the continuous spectrum of $H$. Equation (8.11) shows that one has to have some estimates of the evolution $U(t) P$. Such estimates will be presented in this section. They are enough transparent and can be proved by means of simple (but unfortunately not short) computations which use the spectral resolution of $H$. So let $\vec{h}=P \vec{h}$, then

$$
\begin{gathered}
\|U(t) \vec{h}\|_{\infty} \leq c t^{-1 / 2}\left[\|\vec{h}\|_{2}+N R(\vec{h})\right] ; \\
\|U(t) \vec{h}\|_{\infty} \leq c(1+t)^{-1 / 2}\left[\|\vec{h}\|_{H^{1}}+N R(\vec{h})\right. \\
\|\varrho U(t) \vec{h}\|_{2} \leq c(1+t)^{-3 / 2}\left[\|\vec{h}\|_{2}+N R(\vec{h})\right] .
\end{gathered}
$$

Here

$$
\begin{gathered}
N R(\vec{h}) \text { can be equal }\left\|\left(1+x^{2}\right) \vec{h}\right\|_{1} \text { or }\left\|\left(1+x^{2}\right) \vec{h}\right\|_{2}, \\
\varrho(x)=(1+|x|)^{-\kappa}, \kappa>3,5 .
\end{gathered}
$$

\section{Estimates of nonlinear terms}

All nonlinear terms of euation (8.10) can be estimated with the following set o:

$$
\begin{gathered}
M_{0}(t)=\left|\alpha^{2}-\alpha_{0}^{2}\right|, M_{1}(t)=|d(t)|, d=y-z, \\
M_{2}(t)=\|\vec{\kappa}\|, \vec{\kappa}=\left(\kappa_{1}, \kappa_{2}, \kappa_{3}, \kappa_{4}\right), M_{3}(t)=\|\varrho(z) \vec{h}(z, t)\|_{2}, M_{4}=\|\vec{h}(z, t)\|_{\infty}, \\
\mathbf{M}_{0}(t)=\sup _{\tau \leq t} M_{0}(\tau), b f M_{1}(t)=\sup _{\tau \leq t} M_{1}(\tau), \\
\mathbf{M}_{2}(t)=\sup _{\tau \leq t}(1+\tau)^{3 / 2} M_{2}(\tau), \mathbf{M}_{3}(t)=\sup _{\tau \leq t}(1+\tau)^{3 / 2} M_{3}(\tau),
\end{gathered}
$$




$$
\mathbf{M}_{4}(t)=\sup _{\tau \leq t}(1+\tau)^{1 / 2} M_{4}(\tau)
$$

At last

$$
\mathbf{M}_{j}=\mathbf{M}_{j}\left(t_{1}\right) .
$$

These definitions and relation (7.14) lead more or less directly to the inequalities:

$$
\|\lambda\| \leq W(\mathbf{M})\left(\mathbf{M}_{2}+\mathbf{M}_{3}\right)^{2}(1+t)^{-3}, t<t_{1} .
$$

Here $W(\mathbf{M})$ is a function of $\mathbf{M}_{0}, \mathbf{M}_{1}, \mathbf{M}_{2}, \mathbf{M}_{3}, \mathbf{M}_{4}$, which is a bounded function on a finite vicinity of the point $\mathbf{M}_{j}=0$ and can acquire infinite values outside of some larger vicinity. It is possible to present an explicit expression for $W$ but this expression is useless for our purpose. ¿From (10.1) one can obtain:

$$
\mathbf{M}_{0}, \mathbf{M}_{1} \leq W(\mathbf{M})\left(\mathbf{M}_{2}+\mathbf{M}_{3}\right)^{2} .
$$

Inequalities (10.1) together with the relation (8.7) generate also the estimate

$$
\mathbf{M}_{2} \leq W(\mathbf{M})\left(\mathbf{M}_{2}+\mathbf{M}_{3}\right)^{3} .
$$

Now pick out from $D_{4}$ all terms containing at least one power of $\phi$ and denote their sum by $D_{I I}$, the remainder will be denoted by $D_{I I I}$. Finally, let $D_{I}=$ $D_{1}+D_{2}+D_{3}$.

Direct computations permit to prove the following estimates:

$$
\begin{gathered}
\left\|\left(1+z^{2}\right) P_{1}\left(D_{0}+D_{I}+D_{I I}\right)\right\|_{2} \leq \\
W(\mathbf{M})\left(\mathbf{M}_{2}+\mathbf{M}_{3}+\mathbf{M}_{4}\right)^{2}(1+t)^{-3 / 2} .
\end{gathered}
$$

In order to obtain a similar estimate for $D_{I I I}$ one has to use additionally some information on solutions of nonlinear equation (1.1), more precisely one has to use conservation law (4.3) and estimate (4.5). As result one has obtain:

$$
\left\|P_{1} D_{I I I}\right\|_{2}+\left\|\left(1+z^{2}\right) P_{1} D_{I I I}\right\|_{1} \leq W(\mathbf{M}) \mathbf{M}_{4}^{2 p-1}(1+t)^{-3 / 2} .
$$

Just here it is important to assume that $4 \leq p$.

\section{Final estimates}

Using equation (8.10) and combining estimates (9.1-3),(10.4-5) one can obtain finally:

$$
\mathbf{M}_{3}, \mathbf{M}_{4} \leq W(\mathbf{M})\left[N+\left(\mathbf{M}_{2}+\mathbf{M}_{3}\right)^{2}+\mathbf{M}_{4}^{2}+\mathbf{M}_{4}^{2 p-1}\right],
$$

where

$$
N=\left\|\left(1+x^{2}\right) \chi_{0}\right\|_{2}+\left\|\chi_{0}^{\prime}\right\|_{2}
$$


The first term $\mathrm{N}$ is originated from the first free term of the right side of equation (8.10). It is controlled by the second variants of estimates (9.2-3). Other terms in brackets are originated from the integral term of equation (8.10). They are controlled by estimates (10.4-5) and by both variants of $(9.1),(9.3)$.

Now one has obtained a closed set of inequalities (10.2-3),(11.1) and can try to solve it. Formulas (10.3),(11.1) give the system:

$$
\begin{gathered}
\mathbf{M}_{2}+\mathbf{M}_{3}, \mathbf{M}_{4} \leq \\
W(\mathbf{M})\left[N+\left(\mathbf{M}_{2}+\mathbf{M}_{3}\right)^{2}+\left(\mathbf{M}_{2}+\mathbf{M}_{3}\right)^{3}+\mathbf{M}_{4}^{2}+\mathbf{M}_{4}^{2 p-1}\right] .
\end{gathered}
$$

If $N$ is sufficiently small, the system shows that the pair $\mathbf{M}_{2}+\mathbf{M}_{3}, \mathbf{M}_{4}$ can belong either to a small vicinity of the point $(0,0)$ or to some domain whose distance from $(0,0)$ is limited from below uniformly with respect to $N$. It is clear that only the first possibility can be realized. Therefore all the functions $\mathbf{M}_{0}, \mathbf{M}_{1}, \mathbf{M}_{2}, \mathbf{M}_{3}, \mathbf{M}_{4}$ are sufficiently small:

$$
\mathbf{M}_{j}(t) \leq \mu(N) N,
$$

here $\mu(N)$ is a bounded function defined for small $\mathrm{N}$. Since all constants in the estimates do not depend on $t_{1}$ the same estimates are true for $\mathbf{M}_{j}(t)$ uniformly in $t \in \mathbf{R}_{+}$:

$$
\mathbf{M}_{j}(t) \leq \mu(N) N
$$

\section{The limiting soliton}

Return to (10.1) again. Estimates (11.4) show now that

$$
\|\lambda\| \leq \mu(N) N^{2}(1+t)^{-3} .
$$

It implies that all variables $\gamma, \omega, c, v$ have limits $\gamma_{\infty}, \omega_{\infty}, c_{\infty}, v_{\infty}$ as $t \rightarrow \infty$. So one can introduce the limiting trajectory $\sigma_{+}(t)$ :

$$
\begin{gathered}
\beta_{+}(t)=\omega_{+} t+\gamma_{+}, \omega_{+}=\omega_{\infty}, \gamma_{+}=\gamma_{\infty}+\int_{0}^{\infty}\left(\omega(\tau)-\omega_{\infty}\right) d \tau, \\
b_{+}(t)=v_{+} t+c_{+}, v_{+}=v_{\infty}, c_{+}=c_{\infty}+\int_{0}^{\infty}\left(v(\tau)-v_{\infty}\right) d \tau
\end{gathered}
$$

It is clear that

$$
\sigma(t)-\sigma_{+}(t)=O\left(t^{-1}\right)
$$

as $t \rightarrow \infty$.Now the limiting soliton $w\left(x \mid \sigma_{+}(t)\right)$ arises naturally and

$$
w(x \mid \sigma(t))-w\left(x \mid \sigma_{+}(t)\right)=O\left(t^{-1}\right)
$$

in the space $\mathbf{L}_{2} \cap \mathbf{L}_{\infty}$. 


\section{Dispersion reminder}

The second term $\chi$ of the total solution $\psi=w(x \mid \sigma(t))+\chi$ can be studied asymptotically if one uses the same representantion of $\chi$ as in section 8 ,but with $\infty$ instead of $t_{1}$ in transformation (8.2):

$$
\begin{gathered}
\chi=\exp \left(i \Phi_{\infty}\right) g(z, t), \\
\Phi_{\infty}=-\beta_{+}(t)-\frac{1}{2} v_{+} x, z=x-b(t) .
\end{gathered}
$$

Now the operators $H_{1}$ and $P_{1}$ should be replaced by the naturally defined operators $H_{+}$and $P_{+}$and all construction of section 8-11 can be duplicated.

Particularly one again can separate the contributions of the discrete and the continuous spectra of $H_{+}$:

$$
\vec{g}=\vec{k}+\vec{h}
$$

From

$$
M_{2}(t) \leq \mu(N) N(1+t)^{-3 / 2},
$$

see (11.4), one can obtain at once the estimate:

$$
\vec{k}=O\left(t^{-3 / 2}\right),
$$

in the space $\mathbf{L}_{2} \cap \mathbf{L}_{\infty}$.

Reprezentation (8.10) for $\vec{h}$ acquires the form:

$$
\vec{h}=\exp \left(-i H_{+} t\right) P_{+} \vec{h}_{0}-i \int_{0}^{t} \exp \left[-i H_{+}(t-\tau)\right] P_{+} \vec{D} d \tau,
$$

with the respectively transformed $\mathrm{D}$.

Introduce the representation

$$
\begin{gathered}
\vec{h}=\exp \left(-i H_{+} t\right) \vec{h}_{\infty}+\vec{R}, \\
\vec{h}_{\infty}=P_{+}\left(\vec{h}_{0}+\vec{h}_{1}\right), \vec{h}_{1}=-i \int_{0}^{\infty} \exp \left(i H_{+} \tau\right) \vec{D} d \tau, \\
\vec{R}=-i \int_{t}^{\infty} \exp \left[-i H_{+}(t-\tau)\right] P_{+} \vec{D} d \tau .
\end{gathered}
$$

Here $\vec{h}_{\infty} \in \mathbf{L}_{2} \cap \mathbf{L}_{\infty}: \vec{h}_{0} \in \mathbf{L}_{2} \cap \mathbf{L}_{\infty}$ since $\vec{h}_{0} \in H^{1} ; \vec{h} \in \mathbf{L}_{2} \cap \mathbf{L}_{\infty}$ in accordance with (9.1), (10.4-5). Inequalities (10.4-5) imply immediately that:

$$
\vec{R}=O\left(t^{-1 / 2}\right)
$$

in $\mathbf{L}_{2}$-norm,

$$
\vec{R}=O\left(t^{-1}\right)
$$


in $\mathbf{L}_{\infty}$-norm. So one can formulate the following result:

$$
\begin{gathered}
\psi=w\left(x \mid \sigma_{+}(t)\right)+ \\
+\exp \left(i \Phi_{\infty}\right)\left[\exp \left(-i H_{+} t\right) \vec{h}_{\infty}\right]_{1}(z, t)+R
\end{gathered}
$$

where $R$ admits estimates (13.9-10). The brackets $[\vec{v}]_{1}$ are used in order to indicate the first component of $\mathbf{C}^{2}$ - vector $\vec{v}$.

\section{Scattering}

In the dispersive term $\exp \left(i \Phi_{\infty}\right) \exp \left(-i H_{+} t\right) \vec{h}_{\infty}$ the element $\vec{h}_{\infty}$ belongs to the subspace of the continuous spectrum of the operator $H_{\infty}$. So its behavior as $t \rightarrow \infty$ is scattering behavior (in $\mathbf{L}_{2}$ - norm):

$$
\exp \left(-i H_{+} t\right) \vec{h}_{\infty}=\exp \left(-i H_{0} t\right) \vec{h}_{+}+o(1),
$$

where $\vec{h}_{+} \in \mathbf{L}_{2}$ and is related to $\vec{h}_{\infty}$ in terms of the corresponding wave operator $W_{+}$:

$$
\vec{h}_{\infty}=W_{+} \vec{h}_{+} .
$$

It is not essential that the operator $H_{\infty}$ is not self-adjoint in our case since its spectral resolution has the same structure as for a self-adjoint operator. We are not going to discuss here the properties of $\vec{h}_{+}$and the reminder in more detail. Only note that

$$
\exp \left(i \Phi_{\infty}\left[\exp \left(-i H_{0} t\right) \vec{h}_{+}\right]_{1}(z)=\left[\exp \left(-i l_{0} t\right) f_{+}\right](x),\right.
$$

and

$$
l_{0}=-\partial_{x}^{2}, f_{+}(x)=\exp \left(-i \gamma_{+}-i \frac{1}{2} v_{+} x\right) h_{+}\left(x-c_{+}\right) .
$$

Introduce representation (14.3) in formula (13.11) and write down the final result:

$$
\psi=w\left(\cdot \mid \sigma_{+}(t)\right)+\exp \left(-i l_{0} t\right) f_{+}+o(1) .
$$

From the first author (V.B.):

This work has been written during my stay in the University Paris VII. I want to express my gratitude to A. Boutet de Monvel who was my host and was very helpful in course of this visit.

I am very obliged also to A. Soffer for numerous helpful discussions. 


\section{References}

[Be-Li] Berestycki, H. and Lions, P.L., Nonlinear scalar field equations.I. Existence of a ground state, Arch. Rat. Mech. Anal. 82 (1982), 313345 .

[Ca-Li] Cazenave,T. and Lions,P.L., Orbital stability of standing waves for somenonlinear Schroendinger equations, Comm.Math.Phys. 85 (1982), 549-561.

[G-V] Ginibre,J. and Velo,G., On a class of nonlinear equations. I,II, J Funct. Anal.32(1979),1-71.

[He] Henry,D., Geometrical theory of semilinear parabolic equations, Springer(1981)

[K] Kato,T., On nonlinear Schroedinger equation, Ann.Inst. Henri Poincare, Physique Theorique 46 (1987),113-129.

[Sh-Str] Shatah,J. and Strauss,W., Instability of nonlinear bound states, Comm. Math. Phys.55(1977),149-162.

[Sof-We] Soffer, A. and Weinstein, M.I., Multichannel nonlinear scattering for nonintegrable equations, Comm. Math. Phys.

[Str1] Strauss,W.A., Dispersion of low energy waves for two conservative equations, Arch. Rat. Mech. Anal. 55 (1974), 86-92.

[Str2] Strauss, W.A., Existence of solitary waves in higher dimensions, Comm. Math. Phys. 55 (1977), 149-162.

[We1] Weinstein,M.I., Modulational stability of ground states of nonlinear Shroedinger equations, SIAM J.Math.Anal.16(1985), 472-491.

[We2] Weinstein,M.I., Lyapunov stability of ground states or dispersive evolution equation, Comm. Pure Appl. Math. 39(1981),51-68

V.S.Buslacv

NIIPh, University of St. Petersburg

Peterhof,198904,Russia.

G.S.Perelman

NIIPh,University of St.Petersburg

Peterhof,198904,Russia. 